\title{
Die Dommusik zu St. Stephan im 18. Jahrhundert in sozialgeschichtlicher Perspektive
}

\section{Musical Life at the St. Stephen's Cathedral in Vienna during the 18th Century in Socio-historical Perspective}

Helena Kramářová / helena.kramarova@gmail.com

Department of Musicology, Faculty of Arts, Masaryk University, Brno, CZ

\begin{abstract}
This study deals with the living conditions and certainties of the singers and musicians who served in the Vienna Cathedral of St. Stephen during the 18th century. The focus is on so-called adjunct positions, the occupation of vacant positions and events after the death of a musician. Furthermore, the supply and teaching of choral boys are explained. To the most important changes during the 18th century belonged the new order of worship. These prescriptions of Joseph II influenced the frequency of the figural music performances as well as the financing, which is illustrated by examples.
\end{abstract}

\section{Keywords}

Cathedral St. Stephen, Vienna, music, musicians, Johann Georg Albrechtsberger, Leopold Hofmann, Sterbeqaurtal, Adjunktum, choir boys, social benefits, church reforms 
Die sozialen Sicherheiten, sowie die Stellung der Musiker in der Gesellschaft werden, zusammen mit den aktuellen Tendenzen im Bereich der Alltagsforschung, aus verschiedenen Sichten betrachtet. ${ }^{1}$ Deshalb ist es von Bedeutung, die Abläufe und Vorgehensweisen zu verstehen, die mit der Musikpflege in der Wiener Domkirche zu St. Stephan verbunden waren, da dies ebenfalls Rückschlüsse auf die Verhältnisse in anderen Kirchen ermöglicht. Um diese verstehen zu können, müssen zuerst die Verläufe, die der Kirchenmusik im Stephansdom während des 18. Jahrhundert eigen waren, näher beleuchtet werden.

Die Domkirche befand sich nicht nur unter kirchlicher Aufsicht eines Kirchenmeister, sondern unterlag auch dem Stadtrat, was im 18. Jahrhundert gewisse Abläufe beeinflusste; ${ }^{2}$ dabei handelte es sich hauptsächlich um die Besetzung der freigewordenen Stellen in den Domkapellen, die sozialen Sicherheiten der Musiker, aber auch um die Versorgung der Sängerknaben. Wichtig für die Analyse ist ebenso die finanzielle Lage der Kapellmitglieder, die sich in den 80er Jahren unter Einwirkung der allgemeinen gesellschaftlichen Reformen rasch veränderte. Alle genannten Themen werden, anhand der überlieferten Quellen, genauer erläutert.

\section{Soziale Sicherheiten der Musiker und ihrer Familien}

Die sozialen Sicherheiten, die die Musiker hatten oder nicht hatten werden, ebenso wie die Stellung der Musiker in der Gesellschaft, zusammen mit den aktuellen Tendenzen der Alltagsforschung aus verschiedenen Sichten betrachtet. ${ }^{3}$ Inwiefern die Versorgung der im Stephansdom angestellten Musiker und Sänger einen speziellen Fall darstellte, muss natürlich durch weitere Forschungen in Bezug auf anderen Lokalitäten beleuchtet werden. Trotzdem kann an dieser Stelle behauptet werden, dass manche allgemeinen Tendenzen ebenfalls für diese Kapelle galten.

Zuerst wird näher darauf eingegangen, auf welche Art und Weise die Musiker und Sänger in die Domkapellen eintreten konnten und unter welchen Bedingungen dies üblicherweise geschah. Des Weiteren wird beleuchtet, inwiefern die durch Dekrete angestellten Musiker im Dom tatsächlich musizierten; sowie im Anschluss die Verordnungen über Dienstleistung für hinterbliebene Familien nach verstobenen Musikern.

1 Dieser Text wurde durch das Stipendium AKTION (CZ-AT) ermöglicht. Ein aufrichtiger Dank für zahlreiche Ratschläge gilt Prof. Dr. Otto Biba.

2 Dies ist noch heute ersichtlich, da die Quelle zur Institutionsgeschichte der Dommusik sowohl im Domarchiv als auch im Wiener Stadt- und Landesarchiv vorhanden sind.

3 Dazu siehe: BIBA, Otto. Der Sozialstatus des Musikers, In Joseph Haydn in seiner Zeit. Eisenstadt 1982, S. 105-113; SALMEN, Salmen. Der Sozialstatus des Berufsmusikers vom 17. bis 19. Jahrhundert. Kassel: BärenreiterVerlag, 1971. 


\section{Besetzung der freigewordenen Stellen}

Am häufigsten gibt es im Aktenmaterial verschiedene Unterlagen, in denen die personelle Besetzung der Musikkapelle aufgezeichnet sind. ${ }^{4}$ Anhand dieser überlieferten Dokumente lassen sich für die Domkirche typische Vorgehensweisen bei Besetzung der freien Stellen, Bedeutungen und Funktionen eines Adjunktums, sowie die Voraussetzungen für Eintritte in die Dommusik nachvollziehen.

Ein Todesfall war nicht selten die Grundlage für den Eintritt eines neuen Musikers, da die wenigsten Musiker aus der Domkapelle aus einem anderen Grund austraten. Einer der dies tat, war Urban Möller, der im Jahre 1702 nur das erste Quartalgeld erhielt, weil „umb[!] willen selbiger hernach den geistlichen Stand angetretten“5 hat. Dazu muss betont werden, dass der geistliche Stand an sich kein Hindernis für die Mitwirkung bei der Dommusik war. ${ }^{6}$ Üblicherweise wurde eine Stelle erst nach dem Tod eines Musikers neu besetzt. Die Musiker mussten sich bei dem Kirchenmeisteramt bewerben und der Kirchenmeisteramt gab einen Bescheid an den Magistrat, der die endgültige Entscheidung über Annahme traf. Danach wurde über diese neu besetzte Stelle konferiert und ein Dekret erstellt. Die Entscheidung wurde dann dem Kapellmeister mitgeteilt, wie es beispielsweise im Brief an Johann Georg Albrechtsberger vom 16. Juni 1795 bei der Vergabe der achten Choralistenstelle an Franz Strasser geschah. ${ }^{7}$ Es wurden nicht nur die Stellen in der Essential- und Gnadenbildkapelle neu besetzt, sondern auch die Adjunkten-Stellen. Die Adjunkten-Stellen beim Stephansdom sind vor allem durch das Gesuch um die Adjunkten-Stelle des Komponisten Wolfgang Amadeus Mozart bekannt geworden. ${ }^{8}$ Es gab zwei Arten der Adjunkten; erstens diejenigen, die bereits im Dom festangestellten Musiker zugeteilt wurden, zweitens die, die den Musikern, die im Dom als Aushilfsmusiker tätig waren, unterstellt wurden.

Ein Adjunktum konnte als Bezeichnung für eine zusätzliche Belohnung für einen im Dom etablierten Musiker für bisherige Dienste zuerkannt werden. Im Jahre 1721 wurde der Posaunist Jacob Adalbert Conrad 70 Jahre alt, und war gleichzeitig 42 Jahre lang im Dienst im Stephansdom; deshalb erhielt er von da an jährlich $50 \mathrm{fl}$ zusätzlich. ${ }^{9}$ „Ad vitae dies“ wurden dem zweiten Violinist Fridrich Gött nach 21 Jahren bei St. Stephan 100 fl

4 Die Musiker, die in der Musik zu St. Stephan tätig waren, oder sich um eine Stelle bewarben, wurden im Rahmen des Dissertationsprojekts „Der Kirchenmusikalltag im späten 18. Jahrhundert am Beispiel von Brünn und Wien " erarbeitet, Masaryk-Universität, Brünn, [Einreichtermin: Januar 2020].

5 Wiener Stadt- und Landesarchiv (weiter nur: AT-WStLA), Handschriften, (weiter: HS) A41/20 Kirchenmeisteramts-Rechnungen von St. Stephan - 1702, Fol. 97r.

6 Zu den Geistlichen gehörte z. B. Thomas Guzzo, ein weltlicher Priester und zwischen den Jahren 17431751 angestellte Violinist. Vgl. Domarchiv St. Stephan, Kirchenmeisteramt von St. Stephan (weiter: KMA), Rechnungsbuch 1743, Fol. 110r.

7 Außer Albrechtsberger wurde diese Verordnung auch dem Kirchenmeister und der Buchhaltung mitgeteilt. Vgl. AT-DAWST, KMA, Erledigungen 1792-1800, Kart. 8/2, Fol. 807-808.

8 AT-WStLA, Hauptarchiv, A1/M14, Wolfgang Amadeus Mozart 5, Gesuch um Verleihung der Kapellmeistersadjunktenstelle.

9 AT-DAWST, KMA, Rechnungsbuch 1721, Fol. 56r. 
verliehen, die er bis zu seinem Tod im Jahre 1777 bezog. ${ }^{10}$ Es geschah jedoch relativ selten, dass ein solches Adjunktum vergeben wurde.

Die zweite Art der Adjunkten wurde nach aktuellen Wissenstand unentgeltlich durchgeführt, deshalb ist zu vermuten, dass dazu keine genaueren Angaben vorhanden sind. Dies betrifft jedoch hauptsächlich die Kapellmeister- und Organisten-Adjunkten, die in den Rechnungen kaum zu finden sind. Eine Ausnahme stellt ein Gesuch des dekretierten Organisten-Adjunkts Georg Summer bei der Domkirche aus dem Jahr 1801 dar, aus dem hervorgeht, dass er bereits 21 Jahre in der Domkirche als Organist tätig war; er diente zunächst 16 Jahre unentgeltlich und auch danach wurde er als dekretierter Organist nur gering honoriert (92 $\mathrm{fl}$ pro Jahr). ${ }^{11}$ Wie am Summers Beispiel ersichtlich ist, gab es in der Tat auch Adjunkten-Stellen, die mit einem Adjunktum für geleistete Dienste bezahlt wurden. Hauptsächlich ging es um Tenoristen- und Bassisten-Accessisten, die als zusätzliche Choralisten für Rorate entlohnt wurden. Dazu bekamen einige davon noch ein Adjunktum - eine zusätzliche finanzielle Unterstützung. ${ }^{12}$ Sowohl die bezahlten als auch unbezahlten Adjunkten hatten gemeinsam, dass sie später in der Essential- und/oder Gnadenbildkapelle angestellt wurden. Trotz ungünstiger Bedingungen der Kirchenmusiker ab den 80er Jahren des 18. Jahrhunderts bewarben sich Musiker noch zu Lebzeiten eines angestellten Musikers für die unentgeltlich verrichteten Adjunkten-Stellen, obwohl bereits ein unentgeltlicher Adjunkt als Nachfolger bestätigt worden und im Dom tätig war. Somit konnten sich nicht nur bezahlte Adjunkten, sondern auch unbezahlte an den Musikaufführungen im Dom zu beteiligen.

Die Adjunkten-Stellen hingen eng mit der Fixstellenvergabe zusammen - es war nur durch das Vorrücken möglich, die angestrebte Position in der Musikkapelle zu erreichen. Die Adjunkten bildeten hierbei eine Art Vorstufe beim Vorrücken. Entgeltlich oder unentgeltlich verrichtete Adjunktendienste sollten den Musikern ihren Eintritt in die Domkapelle erleichtern. Die Adjunkt-Stellen können somit als eine Plattform, bzw. Warteliste für künftige Angestellte betrachtet werden, weshalb diese Stellen am häufigsten neu besetzt wurden. Das aus den Quellen ablesbare und in den Gesuchen um Stellen nach dem Tod eines Musikers oft erwähnte Vorrücken ist bereits in den Anfangsjahren des 18. Jahrhunderts zu finden. Die Musiker (besonders die Violinisten) konnten sich nach dem Ableben eines besser besoldeten Kollegen um eine Stelle bewerben und somit die eigene Stellung in der Domkapelle verbessern. Das Vorrücken verlief jedoch nicht zwangsläufig, denn die neue Anordnung musste vom Magistrat bewilligt werden. Eine solche Situation illustriert zum Beispiel die Karriere des Violinisten Anton Fridrich Gött, der im Jahre 1728 seine erste Besoldung als Violinisten-Accessist des zweiten Violinisten Johann Franz Reinhard bekam. Im Jahre 1732, nach dem Tod des ersten Violinisten Franz Timmer, rückte Johann Franz Reinhard an seine Stelle, wodurch Anton

10 AT-DAWST, KMA, Rechnungsbuch 1777, Fol. 92v.

11 Somit wird die Zeitspanne, in der Summer im Stephansdom als Adjunkt tätig war, länger als bis jetzt angenommen, und zwar von 1780-1803. Vgl. AT-DAWST, KMA, Erledigungen 1800-1804, Kart. 9/1, Fol. 1051.

12 In 1720er Jahren wurde als Bassisten-Accessist mit einem Adjunktum von 50 fl. Johann Michael Edlbauer bezahlt. Vgl. AT-DAWST, KMA, Rechnungsbuch 1726, Fol. 57r. 
Fridrich Gött zum secundario Violinist ernannt wurde und seine Bezahlung von $100 \mathrm{fl}$ auf $150 \mathrm{fl} \mathrm{stieg.}{ }^{13}$

In seltenen Fällen konnte eine Beförderung aufgrund der Fähigkeiten und nicht dieser obengenannten Reihung der Musiker durchgeführt werden. So trat zum Beispiel der Posaunist Joseph Hofmann an die Stelle des verstorbenen Bassisten Tobias Gsur. Sowohl der Kapellmeister als auch der Kirchenvater waren damit einverstanden, weil dieser „besagte Herr Hofmann die beste Bassisten-Stimme eines Bassisten hat, und nicht allein auch schon k. und k. Hof Bassist ist, sondern auch sowohl H: Kapellmeister Hoffmann, als auch der dermahlige[!] Kapellmeister Albrechtsberger in der Charwoche zu absingung[!] der Lamentationen demselben gebraucht haben." ${ }^{14}$ Somit konnte der zweite Posaunist auf die freigewordene Bassistenstelle vorrücken.

Was das Beenden der Dienste bei der Dommusik angeht, war die Kündigung eines Musikers seitens des Magistrats nicht üblich - dies galt nicht nur für die Dommusiker, sondern auch für Musiker die in anderen, kleineren Kirchen tätig waren. Sogar eine schwere Krankheit oder hohes Alter war kein Grund, um einen Musiker zu kündigen. ${ }^{15}$ Wenn ein Musiker oder Sänger nicht mehr in der Lage war, sein Dienst zu leisten, wurde ein Adjunkt oder Accessisst beauftragt, ihn zu vertreten. Sofern sich ein Musiker also nicht selbst entschied die Kapelle zu verlassen, war seine Stelle fast ohne Ausnahmen bis zu seinem Ableben gesichert. Umgekehrt jedoch waren ein nicht allzu hohes Alter und ein guter gesundheitlicher Zustand Voraussetzungen für die Anstellung eines Musikers.

Diese beiden Voraussetzungen werden durch die Korrespondenz zwischen dem Posaunisten Jacob Moser und dem Wiener Magistrat veranschaulicht. Moser versuchte mehrmals eine Zusage einer Accessisten-Stelle vom Magistrat zu erhalten. Er wies mehrmals darauf hin, dass der tatsächliche Kornettist sehr alt und kränklich sei, ebenso wie sein 82-jähriger Adjunkt Klemens Messerer, der längst nervenschwach sein solle. Nach zwei Jahren wiederholt er seine Bitte um dieselbe Position, die wieder abgewiesen wurde. Als Begründung wurden jedoch Alter und gesundheitlicher Zustand des Bittstellers betont, sowie dass er seinem Instrument nicht mehr gewachsen sei. ${ }^{16}$ Daraus ergibt sich, dass die jüngeren und gesunden Musiker bei der Aufnahme in die Domkapelle bevorzugt wurden, wenn sie aber während ihrer Dienste erkrankten, wurden sie nicht gekündigt und weiter bezahlt.

\section{Anstellungskumulation und Vertretungsmöglichkeiten}

Es überrascht wenig, dass die meisten Kirchenmusiker aus den Domkapellen nicht nur in der Domkirche zu St. Stephan tätig waren, sondern auch in der Hofkapelle oder

13 AT-DAWST, KMA, Rechnungsbuch 1732, Fol. 59r.

14 AT-DAWST, KMA, Erledigungen 1792-1800, Kart. 8/1, Fol. 761; diese Vorgehensweise ist ebenfalls in den Rechnungsbüchern aus dem Jahr 1794 zu finden. Vgl. AT-DAWST, KMA, Rechnungsbuch 1794, S. 64.

15 Vgl. SEHNAL, Jiří. Hudba v olomoucké katedrále v 17. a 18. století. Brno: Moravské muzeum, 1988.

16 AT-DAWST, KMA, Erledigungen 1804-1808, Kart. 10/1, Fol. 1272. 
anderen Kirchen Wiens dienten. Hierbei war es nicht unüblich, dass die Dommusiker, anstatt diesen Dienst selbst zu verrichten, andere Musiker beauftragten, und diese direkt von ihrem eigenen Gehalt bezahlten. Diese nicht konferierten Musiker sind dadurch nur teilweise namentlich bekannt, denn ihre Tätigkeit wurde oft nicht dem Magistrat oder der Kirchenverwaltung bekannt gegeben. Mithilfe drei konkreter Beispiele werden nun diese Problematik und die dazugehörige Stellungnahme des Magistrats veranschaulichet.

Allgemein betrachtet stellte die Besetzung der verschiedenen Stellen in mehreren Musikensembles solange kein Problem dar, bis diese die Qualität der Musik bei St. Stephan beeinflusste. Die Anstellung in der Hofkapelle und die daraus folgenden Pflichten zählten zu den Ämtern, die durch Vertretungen besetzt und geregelt werden konnten. Dies illustriert der Briefwechsel aus dem Jahr 1793 nach dem Tod des zweiten Choralisten $^{17}$ Martin Leopold Grebner, der aber in den Rechnungen aus den 80er Jahren ausschließlich als zweiter Violonist bezeichnet wurde und in den 90er Jahren in den offiziell geführten Angaben der Buchhaltung nicht mehr vorkommt. Nichtsdestotrotz endete seine Tätigkeit bei der Dommusik erst mit seinem Tod. Das belegt ein Bittschreiben von Anton Kayßler, der um Grebners Choralisten-Stelle bat.

Obwohl Grebner nicht als Choralist durch ein Dekret angestellt war, übernahm er einige Pflichten des ersten Choralisten (bzw. Subcantor) Joseph Krottendorfer. Als erster Choralist bekam Joseph Krottendorfer ein jährliches Gehalt von $200 \mathrm{fl}$. Gleichzeitig war er aber in der Hofkapelle beschäftigt, deshalb bat Krottendorfer den Magistrat um Erlaubnis, „[...] H: Grebner alß seinen Substituten gegen jährlich $40 \mathrm{fl}$, welche er von seiner Besoldung wollte fahren lassen, zu nehmen, damit, wan[!] er etwa bey Hof zu thun hätte, und verhindert wurde seinen Choralistendienst bey St. Stephan abzuwarten, H: Grebner an statt[!] seiner diese dienste[!] verstehe. " ${ }^{18}$ Diese Vertretung wurde vom Wiener Magistrat bewilligt. Aufgrund dessen sollte die Entscheidung von Joseph Krottendorfer getroffen werden, ob Kayßler diese Choraldienste anstatt des Choralisten Grebner übernehmen solle. Krottendorfers Zustimmung wurde dem Magistrat schriftlich bekannt gegeben und Anton Kayßler konnte diesen Dienst übernehmen. ${ }^{19}$

Eine auf den ersten Blick geläufige Bitte um eine finanzielle Beihilfe gibt Auskunft über andere Substituten, die zusätzliche Informationen über interne Vorgänge bringt. Für sieben Jahre und vier Monate sollte Franz Mosbrucker als substituierender Organist bei St. Stephan dienen, und dafür ein monatliches Gehalt von $9 \mathrm{fl}$ bekommen haben. Anhand seines Briefes sollte er an Jahrestagen das tägliche Choralamt und die Segenmesse begleiten, am Nachmittag dann um 16 Uhr die Choralvesper. Durch diese beschwerliche Dienstleistung habe er sich Lungenschwäche zugezogen, was ein Grund war, weshalb er sich an den Magistrat wandte: sein Arzt empfahl ihm eine medizinische Kur, die er sich aber nicht leisten könne. ${ }^{20}$

17 Ab 1784 bezeichnete die Benennung „Choralist" die Personen, die für Choraldienste verantwortlich waren. Es handelte sich nicht mehr um Bezeichnung der Sänger - sie wurden seitdem ausschließlich anhand ihrer Stimmlage tituliert (Tenorist, Bassist, etc.).

18 AT-DAWST, KMA, Erledigungen 1792-1800, Kart. 8/1, Fol. 727.

19 Vgl. Ebenda, Fol. 727.

20 AT-DAWST, KMA, Erledigungen 1800-1804, Kart. 9/1, Fol. 1100. 
Aus der Antwort des Magistrats geht hervor, dass sich der Bittsteller zwar als Organist bei der Domkirche zu St. Stephan vorstellt und ausweist, er dem Magistrat aber unbekannt war. Nach durchgeführter Untersuchung zeigte sich, dass er ohne Wissen des Magistrats als substituierender Organist tätig war. Dem Magistrat missfiel die Sache, weil die Dienste unter den eigentlichen Organisten Matthäus Mittlmayer und dessen Adjunkt Georg Summer hätten ordnungsgemäß aufgeteilt werden sollen, weshalb kein substituierender dritter Organist nötig gewesen wäre. Deshalb sollte Mosbrucker den Zuschlag mit dem offiziell substituierten Organisten Mittlmayer klären - seine Bitte wurde abgelehnt. ${ }^{21}$

$\mathrm{Zu}$ einer weiteren Beschwerde des Magistrats über die nicht erlaubte Vertretung kam es beim Stellengesuch von oben erwähnten Posaunisten Jacob Moser. Durch das Hervorheben seiner Substitution erhoffte er sich wahrscheinlich, Vorzüge im Annahmeverfahren zu erhalten, da er seine Fähigkeiten bereits als Substitut unter Beweis gestellt habe. Der Reaktion nach erzielte er das Gegenteil. Der Kirchenmeister Sigel schrieb, dass ,es immer für die Kirchenmusik von St. Stephan traurig und nachsichtig [war], dass die wirklich besoldeten Individuen persönlich selten Dienste sein und willkürlich Substituten schickten. "22 Diese Substituten wurden durch die eigentlich angestellten Musiker von deren eigenem Lohn bezahlt, weshalb die tatsächliche Besetzung der Musiker in der Domkapelle ständig variierte und heute nicht mehr genau nachvollziehbar ist.

Die angeführten Beispiele bezeugen, dass dem Magistrat die verschiedenen Vertretungen der Angestellten zwar allgemein bekannt waren, trotzdem wussten die Verwalter nicht in allen Fällen um die tatsächliche Identität der Sänger und Musiker, was zur Unzufriedenheit führte.

\section{Sterbequartal für Witwen und die Ehre Gottes}

Im breiteren Kontext der Sozialsicherheiten von Kirchenmusikern des 18. Jahrhunderts scheint das sogenannte Sterbequartal besonders außergewöhnlich sein. Die durch ein Dekret gesicherte finanzielle Unterstützung wurde den hinterlassenen Witwen, bzw. den Erben, nach dem Tod eines regulär angestellten Musikers gegeben. Im Dekret vom 23. Juni 1722 wird die Vorgehensweise bei einem Sterbefall von Musikern der Dommusik festgelegt. Vom Sterbedatum an wird den Hinterbliebenen die Auszahlung eines Quartalsgehaltes - immer in der Höhe des Quartalsgehalts zu Lebzeiten des jeweiligen Musikers - zugesichert. Somit kann das Sterbequartal als eine Sozialleistung betrachtet werden, die die schwierige Situation der Musikerfamilien wenigstens temporär erleichtern sollte. ${ }^{23}$

21 Vgl. Ebenda, Fol. 1100.

22 AT-DAWST, KMA, Erledigungen 1804-1808, Kart. 10/1, Fol. 1272.

23 Die soziale Absicherung von Musikern wie Angehörigen war eine der Aufgaben der Tonkünstler-Societät, die als Pensionsverein für Witwen und Waisen österreichischer Tonkünstler im Jahr 1771 gegründet wurde. Vgl. HILSCHER, Elisabeth Th. Wiener Tonkünstler-Sozietät. In Oesterreichisches Musiklexikon online [online], 2001. [cit. 2019-05-13] URL: 〈https://www.musiklexikon.ac.at/ml/musik_T/Tonkuenstlersozietaet.xml>. 
Die Auszahlung verzögerte das Vorrücken der Musiker um diese angegebene Zeit, somit sind die Ausgaben für die Musik bei St. Stephan aufgrund eines Todesfalls bzw. der Auszahlung des Sterbequartals nur selten gestiegen.

Das erste Sterbequartal wurde bereits 1722 an Maria Theresia Römerin gezahlt, die Witwe des verstorbenen Domorganisten Anton Römer. ${ }^{24}$ Seitdem wurde das Sterbequartal regelmäßig bei allen Todesfällen der Mitglieder sowohl der Essential- als auch Gnadenbildkapelle an die Angehörige ausgezahlt. Zu einer schriftlichen Beschwerde kam es am 22. November 1800. Das gesamte Musikpersonal wandte sich an das Wiener Magistrat mit der Bitte um die Bestätigung und Gewährleistung des Sterbequartals für die Witwen und die hinterbliebenen Erben mit der Berufung auf die bereits 50 [sic!] Jahre bestehende Konvention..$^{25}$ Grundlage für diese Beschwerde war die Tatsache, dass es öfters passiert sein soll, dass das Quartalgeld nicht der Witwe übergeben wurde, sondern in der Kirchenkasse behalten wurde, bis ein neuer Musiker anstatt des Verstorbenen bestätigt war. Das Musikpersonal berief sich an die Gerechtigkeit des Magistrats und wies darauf hin, dass solche Tatsachen nicht zur Ehre Gottes beitragen.

Der Magistrat äußerte sich dazu ausführlich, und zwar, dass ihm die Existenz eines solchen Dekrets unbekannt sei, und daher müsse es sich um eine Konvention aus früheren, reicheren Jahren handeln. Was die Ehre Gottes angeht, wurde dem gesamten Musikpersonal vom Magistrat empfohlen, dass „,...] sie am ersten brauchen würden, kein so elendes Zeug in der Musik bey abhaltenden Gottesdiensten zu machen, damit einmahl[!] der üble, und für die Kirchenvorsteher äusserst nachseilige Ruf hinweggewälzet würde, dass das ganze Wiener Publikum nicht mehr sagen darf, dass bey St. Stephan die schlechteste Musik seyn, die doch der Kirche ein so enormes Geld kostet; [...] "26 Falls das Niveau des Musizierens sich nicht verbessere, drohte er, dass der Musik ein oder mehrere Subjekte entzogen werden können und die Anzahl der Angestellten aufgrund von Ersparnissen reduziert werde, obwohl die Musik darunter leiden könne. Wenn sie selbst ordentlich zum Gottesdienst kämen und nicht wie bisher bei jedem vierten bis sechsten, oder aber auch gleich bei mehreren, ausblieben, könnte der schlechte Ruf wieder verbessert werden. Dazu wurde hinzugefügt, dass diese Entscheidung in den Händen der Musiker läge. Somit betrachtete das Magistrat die Bitte als erledigt; die Vorgesetzten hatten nichts dagegen, wenn den Musikpersonal unter sich das Sterbequartal verrichten würde. Sekundär bezeugt diese Aussage von der Seite des Magistrats die allgemeine Unzufriedenheit mit dem Musikbetrieb im Stephansdom. Ob die beschriebene Situation der Wirklichkeit entsprach, oder der schlechte Ruf nur als Argument benutzt wurde, lässt sich nicht eindeutig beweisen. Trotzdem wurde dadurch eine zeitgenössische Reflexion des Musikgeschehens um die Wende des Jahrhunderts überliefert.

Die Bittsteller wiederholten das Gesuch und als Anhang fügten sie nicht nur das Original des Ratschlags vom 23. Juni 1722, sondern noch zwei Kopien hinzu. ${ }^{27}$ Ihre For-

24 Vgl. AT-DAWST, KMA, Rechnungsbuch 1722, Fol. 59r.

25 AT-DAWST, KMA, Erledigungen 1800-1804, Kart. 9/1, Fol. 1005.

26 Ebenda, Fol. 1005.

27 AT-DAWST, KMA, Erledigungen 1804-1808, Kart. 10/1, Fol. 1202. 
derung blieb gleich - sie wollten dadurch die Bestätigung der bestehenden Konvention um Ansuchen des Sterbequartals erzielen. Angesichts des aufgetauchten Dekrets wurde unmöglich - egal ob sich die oben angedeuteten Verhältnisse bei den Musikproduktionen verbesserten oder nicht, den Hinterbliebenen das Sterbequartal zu verweigern. Die Verordnung wurde schriftlich bestätigt, nämlich „von dem Tage des Todes ein vierteljähriger Genuß sowohl der Besoldung, als auch gesammten Accidenten um anhängige Emolumente zur Grad und Abfertigung zuständig sey, mithin der in den Dienst eintretende Musicus durch viertjährige Zeit der Besoldung, accidentien[!] und übrigen Emolumenten gänzlich cariren solle."28

Das Sterbequartal wurde von da an wieder ausbezahlt; unter den Akten lassen sich die verschiedenen Gesuche nach dem Tod von Musikern finden. Im Jahr 1803 bat zum Beispiel die Witwe Adlmanns darum;:29 1806 wurde das Sterbequartal an die Hinterbliebenen des verstorbenen Karl Ponheimer ${ }^{30}$ und Joseph Kaukol ausbezahlt. ${ }^{31}$ Im Jahre 1809 starben gleich drei wichtige Persönlichkeiten der Domkapelle - nach dem Tod des ersten Violinisten Joseph Hofmann bekam seine Ehefrau das Sterbequartal, ${ }^{32}$ danach folgte ein Gesuch von Rosalia Albrechtsberger, Witwe des Kapellmeisters Johann Georg Albrechtsberger. ${ }^{33}$ Im Juli verlangten die Kinder des Domorganisten Georg Summer das ihnen zustehende Geld. ${ }^{34}$ Bei dem letztgenannten Antrag wurde als Anhang Summers Organisten-Dekret und Abschrift der Sperrrelation seines Nachlasses beigelegt. Die Krankheits- und Begräbniskosten ( $378 \mathrm{fl} 40 \mathrm{x}$ ) bezahlte seine Tochter Antonia, die sich während seiner Krankheit um ihn kümmerte, deshalb bekam sie seinen Nachlass in der Höhe von $307 \mathrm{fl} 30 \mathrm{x}$, um diese Unkosten zu tilgen. Das Sterbequartal stellte mit 162 fl 30 x eine Möglichkeit dar, mit der die dem Tod verbundene Kosten, wie zum Beispiel die Bestattungskosten, den Hinterbliebenen abzunehmen.

Die Besetzung der freigewordenen Stellen durch, meistens unentgeltlich agierende, Adjunkten kann als gewisse Qualitätssicherung bei der Auswahl der Sänger und Musiker betrachtet werden, sowie damit auch gleichzeitig das starke Interesse der Musiker an der festen Anstellung bei St. Stephan bezeuget wird. Nachdem Musiker Mitglieder der Dommusik wurden, sorgten sowohl Magistrat als auch Kirchenmeister aufgrund bestehender Traditionen und Verpflichtungen für sie. Durch das Vorrücken konnten sie ihre finanzielle Lage verbessern und ab 1722 wurde nach ihrem Tod auch das Einkommen der Musikerfamilien wenigstens für ein Vierteljahr gesichert.

28 Ebenda, Fol. 1202.

29 Vgl. AT-DAWST, KMA, Erledigungen 1800-1804, Kart. 9/2, Fol. 1146.

30 Vgl. AT-DAWST, KMA, Erledigungen 1804-1808, Kart. 10/2, Fol. 1304.

31 Vgl. AT-DAWST, KMA, Erledigungen 1804-1808, Kart. 10/2, Fol. 1316.

32 Vgl. AT-DAWST, KMA, Erledigungen 1808-1811, Kart. 11/1, Fol. 1461.

33 Vgl. AT-DAWST, KMA, Erledigungen 1808-1811, Kart. 11/1, Fol. 1446.

34 Vgl. AT-DAWST, KMA, Erledigungen 1808-1811, Kart. 11/1, Fol. 1453. 


\section{Sängerknabeninstitution}

Die Domkapelle hatte, sowie andere kirchliche Institutionen auch, eigene Sängerknaben, die zusammen mit den erwachsenen Sängern und Musikern mitwirkten. Der Magistrat stellte nicht nur Mittel für ihre Versorgung zur Verfügung, sondern kümmerte sich um ihre Ausbildung.

\section{Knabenversorgung}

Am Anfang des 18. Jahrhunderts hatte der Kapellmeister sechs Kapellknaben zur Verfügung. In den früheren Jahren standen drei unter Aufsicht des Kapellmeisters und die anderen drei wurden vom Organisten, bzw. dem Kapellmeister der Gnadenbildkapelle versorgt. Für diese Versorgung wurde sogenanntes Kostgeld ausbezahlt. Aus den Rechnungsbüchern 1702 und 1709 gehen die Teilbereiche hervor, aus denen sich während des 18. Jahrhunderts das Kostgeld für Knaben zusammensetzte, nämlich ein Präzeptor, Waschgeld, Ausgaben für Arzt und Medikamente, sowie ein Barbier. Bis ins Jahr 1721 wurde auch Geld für den Knabenunterricht - Komposition und Instruktion - inkludiert.

Ab dem Jahr 1716, in dem Georg Reutter d. Ä. Kapellmeister wurde, wurden alle Unterhaltskosten für die Knaben ausschließlich an den Domkapellmeister ausgezahlt. Er bekam jährlich $1200 \mathrm{fl}$ als Kostgeld, im Jahre $1741 \mathrm{kam}$ dazu „zugelegte Adjutum[!] auf das Kostgeld. " ${ }^{35}$ Bereits im Jahre 1747 wurde zusätzlich ein Zuschlag bewilligt. Laut Relation Nr. 238 wurde dem Kapellmeister künftig die Zulage von 200 fl jährlich zu den bestehenden 100 fl versichert. ${ }^{36}$ Am 12. Juli 1775 kam dazu unter Leopold Hofmann noch eine spezielle Zulage von $300 \mathrm{fl}$ jährlich. Dies veränderte sich nach der Musikregulierung im Jahre 1784. Ab 1785 wurde jährlich das Kostgeld mit 1500 fl, mit einem Zuschlag von $300 \mathrm{fl}$, festgelegt.

Erst seit dem 1. April 1793 durfte die Anzahl von sechs auf acht erhöht werden. Seitdem unterhielt der Kapellmeister Albrechtsberger acht Sängerknaben. ${ }^{37}$ Ihm wurde in Folge dessen vom Magistrat am 1. August $300 \mathrm{fl}$ jährlich zugelegt.

\section{Ausbildung der Knaben}

Außer Kostgeld bekam der Kapellmeister ab 1721 jährlich $75 \mathrm{fl}$ als sog. „Instructionsgeld“, wobei der Kapellmeister selbst die Verantwortung für den Unterricht trug. Dies änderte

35 Da das Jahr 1741 nicht mehr vorhanden ist, wird diese Tatsache zum ersten Mal im Rechnungsbuch aus dem Jahre 1743 mit dem Hinweis auf 1741 vermerkt. Vgl. AT-DAWST, KMA, Rechnungsbuch 1743, Fol. 104v.

36 Vgl. AT-DAWST, KMA, Rechnungsbuch 1747, Fol. 80v.

37 Vgl. AT-DAWST, KMA, Erledigungen 1792-1800, Kart. 8/1, Fol. 759. 
sich erst im Jahr 1779, als es zu einer Unterrichtsumstrukturierung kam. Der Kapellmeister Hofmann beschrieb den Unterrichtszustand als unzureichend. Die Kapellknaben sollten Anspruch auf einen Normallehrer (jährlich $72 \mathrm{fl}$ ), dazu monatlich $6 \mathrm{fl}$ für einen Schlagmeister und weitere $6 \mathrm{fl}$ für einen Geigenmeister haben, wofür der Organist und der erste Geiger bei St. Stephan zur Verfügung standen. Überdies sollten sie ebenfalls im Gesang unterrichtet werden, obwohl man Hofmanns Brief nach meinte, dass dies nicht notwendig sei, da die Knaben bereits beim Eintritt in das Kapellhaus ohnehin schon singen können müssten. Weiters befand man, dass die älteren Knaben die jüngeren, neu gekommenen, unterrichten könnten, damit keine weiteren Unkosten für den Kapellmeister entstehen. Hofmann war aber streng dagegen; seines Erachtens brachte dies keine Vorteile und er meinte, dass ein Gesangmeister die Knaben im Gesang unterrichten solle. Der Gesangunterricht sollte täglich im Kapellhaus stattfinden. Einer der wichtigsten Gründe, weshalb Unterricht für die Knaben unentbehrlich sei, war laut Hofmann, dass sie ,theils vom Lande herein, theils gar von anderen Ländern, als Mähren, Böhmen, und Schlessien herkommen, eine ganz andere Solmisation gewöhnt sind, und weder unsere hiesige Musique Art im Singen, noch in der Aussprache haben." ${ }^{88}$ Deshalb schlug Leopold Hofmann folgende Lösung vor: die zwei genannten Meister sollten nur $4 \mathrm{fl}$ monatlich erhalten, und vom restlichen Geld sollte der Gesangmeister bezahlt werden. Somit könnte die Jugend einen weiteren Musikmeister und Musikunterricht erhalten, ohne die Kosten zu erhöhen. Ob seine Vorschläge akzeptiert wurden, geht aus diesem Brief nicht hervor.

Aus den Kirchenmeisteramts-Rechnungen kann die Situation besser nachvollzogen werden. Im März 1779 wurde eine Resolution erlassen, in der die Vergütung der einzelnen Lehrer verankert wurde, sowie es Hofmann bereits in seiner Bitte schrieb. Unter den Besoldeten erschien 1784 zum ersten Mal ein Singmeister, d.h. erst fünf Jahre nach Hofmanns Bitteschreiben.

Die Knabenlehrer genossen bis ins Jahr 1808 die gleiche Besoldung. Am 1. August 1808 stellte der Kapellmeister Georg Albrechtsberger einen Antrag um ihre Gehaltvermehrung. ${ }^{39}$ Er führte an, dass jeder Musiklehrer nur $48 \mathrm{fl} \mathrm{jährlich} \mathrm{erhielt,} \mathrm{was} \mathrm{zwar} \mathrm{eine}$ Zeit lang angemessen war, aber "gegenwärtig kein Lehrer der Musik zu bekommen ist, welcher Talent und Fleiß genug zu diesem mühsamen Geschäft besitzt. ${ }^{"}{ }^{0}$ Er bat daher, um eine den damaligen Zeiten angemessene Zulage für die drei Lehrer. Albrechtsbergers Erachten nach könnten sich aus der Gehaltserhöhung einige Vorteile ergeben. Wenn ein guter Musiker die Sängerknaben unterrichten würde, wäre dies deutlich besser, als wenn „die Zöglinge kein halb virtuosen, oder gar Stümpler[!] unterrichtet. ${ }^{\text {"11 }}$ Die bisherigen Fortschritte der Kinder und ihre Pflichterfüllung sollten das beste Zeugnis sein. Wenn jedoch die Qualität des Unterrichts sinken würde, fürchtete Albrechtsberger, dass kein Knabe mehr in St. Stephan eintreten wolle. Vor allem, weil die Sängerknaben bei dem Austritt aus dem Kapellhaus weder eine Remuneration noch ein Stipendium bekamen - somit

38 AT-DAWST, KMA, Erledigungen 1771-1786, Kart. 7/2, Fol. 402.

39 AT-DAWST, KMA, Erledigungen 1808-1811, Kart. 11/1, Fol. 1450.

40 Ebenda, Fol. 1450.

41 Ebenda, Fol. 1450. 
stellen die erworbenen Fähigkeiten er das Einzige dar, was sie als eine Investition in die Zukunft mit sich mitnehmen konnten.

\section{Auswirkungen der Gottesdienstreform Kaiser Josephs II. und Re- form der Dommusik}

Das wichtigste Ereignis für die Kirchenmusik und Kirchenmusiker war die Gottesdienstreform, die von Kaiser Joseph II. verordnet wurde. Seine Verordnungen und Reformen betrafen vor allem die Gottesdienstordnung, sowie die Existenz der Bruderschaften und Restriktionen der Andachten. ${ }^{42}$ Diese durchgeführten Reformen hatte starke Auswirkungen auf den Musikeralltag beim Stephansdom, welche zwar sehr maßgeblich waren, jedoch für die Dommusik bei St. Stephan nicht unbedingt als ,fatal' zu bezeichnen sind. Anhand der Quellen wird gezeigt, inwiefern die Dommusik betroffen war und zu welchen notwendigen Veränderungen es kommen musste.

Bevor auf Einzelfälle eingegangen werden kann, müssen die wichtigsten Umwandlungen der Domkapelle erwähnt werden. Die erste erhaltene Nachricht betreffend der Besoldungen findet sich im Brief vom 24. Januar 1784, in dem darauf hingewiesen wurde, „daß mit Ende [Dezem]bris [1]783 die Musik Besoldungen bey der St: Stephans Kirche bis auf weitere Regulierung derenselben[!] aufgehoben werden sollen, "43 woraufhin laut des Verfassers anzunehmen war, dass die bisher empfangenden Entlohnungen der Musiker von der Corporis-Christi-Bruderschaft ein Ende nahmen und die Musiker deshalb nur für die verbleibenden Dienste besoldet wurden. ${ }^{44}$

\section{Besoldungen vor und nach der Musikreform im Vergleich}

Es stellt sich nun die Frage, wie sich die Gehälter der Dommusiker entwickelten. In den untenstehenden Tabellen wird veranschaulicht, wie die einzelnen Besoldungen aussahen. Zu betonen ist hierbei, dass die Löhne der Choralisten, sowie die der Posaunisten, das ganze Jahrhundert hindurch bis zu der Musikreform gleichblieben. Es darf jedoch nicht vergessen werden, dass ein nicht unbedeutender Bestandteil der Choralistenbesoldung Deputate darstellten, die separat bezahlt wurden und sich in den offiziellen

42 Mit den Auswirkungen der Reformen auf den Musikbetrieb in Wien setzte sich Otto Biba auseinander. In den von ihm behandelten Akten fehlen jegliche Angaben zur Musikkapelle zu St. Stephan, denn die Domkirche unterlag dem Stadtrat. Vgl. BIBA, Otto. Die Wiener Kirchenmusik um 1783. In Beiträge zur Musikgeschichte des 18. Jahrhunderts (I/2). Eisenstadt: Institut für Österreichische Kulturgeschichte, 1971, S. 7-67; auf diese Tatsache wies neulich Elisabeth Hilscher hin, Vgl. HILSCHER, Elisabeth. Musikbruderschaften als Karrierenetzwerke für Stadt und Hof in Wien. In LOBENWEIN, Elisabeth - SCHEUTZ, Martin - WEISS, Alfred Stefan (Hg.). Bruderschaften als multifunktionale Dienstleister der Frühen Neuzeit in Zentraleuropa. Wien: Böhlau, 2018, S. 364.

43 AT-DAWST, KMA, Erledigungen 1771-1786, Kart. 7/2, Fol. 442.

44 Vgl. Ebenda, Fol. 442. 
Verzeichnissen nicht finden lassen. Die Senkung der Deputate war eines der größten Probleme, die in Bezug auf die Finanzierung der Musik nach der neuen Pfarrverteilung entstanden sind. In der Stadtpfarrkirche zu St. Jakob in Brünn betrug das Einkommen von den Deputaten das Dreifache ihres Gehaltes (55 fl 38 x vs. 158 fl 40 x). ${ }^{45}$ Ähnlich durfte auch die Situation in der Domkirche ausgesehen haben.

Im Laufe des Jahrhunderts stieg jedoch nicht nur die Anzahl der Violinisten, sondern auch die Lohnhöhe, wie in folgender Tabelle ${ }^{46}$ anhand der ausgewählten Jahre abzulesen ist:

\begin{tabular}{|l|l|l|l|l|}
\hline & $\mathbf{1 7 1 2}$ & $\mathbf{1 7 3 6}$ & $\mathbf{1 7 5 3}$ & $\mathbf{1 7 7 6}$ \\
\hline 1. Violinist & $200 \mathrm{fl}$ & $225 \mathrm{fl}$ & $250 \mathrm{fl}$ & $250 \mathrm{fl}$ \\
\hline 2. Violinist & $100 \mathrm{fl}$ & $150 \mathrm{fl}$ & $150 \mathrm{fl}$ & $150 \mathrm{fl}$ \\
\hline$[$ sine] Violinist & $100 \mathrm{fl}$ & $150 \mathrm{fl}$ & $150 \mathrm{fl}$ & - \\
\hline$[$ sine] Violinist & $100 \mathrm{fl}$ & $100 \mathrm{fl}$ & $225 \mathrm{fl}$ & $225 \mathrm{fl}$ \\
\hline$[$ sine] Violinist & - & $100 \mathrm{fl}$ & $225 \mathrm{fl}$ & $225 \mathrm{fl}$ \\
\hline$[$ sine] Violinist & - & - & $100 \mathrm{fl}$ & $100 \mathrm{fl}$ \\
\hline$[$ sine] Violinist & - & - & $100 \mathrm{fl}$ & $104 \mathrm{fl}$ \\
\hline$[$ sine] Violinist & - & - & $50 \mathrm{fl}$ & $40 \mathrm{fl}$ \\
\hline$[$ sine] Violinist & - & - & $50 \mathrm{fl}$ & - \\
\hline Vestenburgl: Violinist & - & - & $40 \mathrm{fl}$ & $32 \mathrm{fl}$ \\
\hline Vestenburgl: Violinist & - & - & $40 \mathrm{fl}$ & $32 \mathrm{fl}$ \\
\hline Violinist (Stiftungsüberschuss) & - & - & - & $19 \mathrm{fl} 12 \mathrm{x}$ \\
\hline
\end{tabular}

Da die einzelnen Jahrgänge der Rechnungsbücher aus den 80er Jahren erhalten geblieben sind, lassen sich die Veränderungen sowie die Auswirkungen der Reform bei den offiziell zuerkannten Gehältern gut nachvollziehen. Die Gehälter des gesamten Musikpersonals wurden durch das Dekret Nr. 533 vom 11. Mai 1784 neu reguliert. ${ }^{47} 1784$ wurden die Musiker zuerst für das erste Quartal nach der vorherigen Tradition besoldet, ab dem zweiten Quartal galt die neu verordnete Lohnhöhe. Überdies mussten noch die Sterbequartale für den Bassisten Ferdinand Schallhaß, den Posaunisten Leopold Christian und den Fagottisten Jacob Payer ausbezahlt werden, sowie die ausständige GnadenbildBesoldung $^{48}$ von $29 \mathrm{fl} 4$ x für den Posaunisten Johann Adlmann. Aus diesen Gründen werden an dieser Stelle die Jahre 1783 und 1785 verglichen.

45 Vgl. Archiv města Brna (AMB) [Archiv der Stadt Brünn], Bestand A1/12 Spisovna in publicis sine signo, Inv.-Nr. 3023, sine Fol. Plan / Einer Verhältnußmessigen Eintheilung zwischen / den allerhöchst bestimmten drey Pfarreyen der / k. Haupt Stadt Brünn.

46 Ausgewählt wurden Jahre, in denen keine Sterbequartale zu zahlen waren, und die somit nur reguläre Ausgaben abbilden. Die Violinisten wurden, außer den ersten zweien, auch nicht nummeriert dargestellt, da es bis in die 80er Gewohnheit war, nur die Angaben bei den primario und secundario Violinisten zu vermerken.

47 Vgl. AT-DAWST, KMA, Rechnungsbuch 1784, Fol. 84r.

48 Dieser Eintrag wurde in der Rubrik Auf die Vokal- und Instrumentalmusik angegeben, obwohl die Rubrik Auf die Musik alda [Gnadenbildkapelle] zur Verfügung steht. Dort befindet sich nur die Angabe über Ausgaben „Nichts.“ Vgl. HOCHRADNER, Thomas - VÖSÖMARTY, Géza Michael. Zur Musikpflege am Altar Mária Pócs (Maria Pötsch) in St. Stephan in Wien. In Studia Musicologica Academiae Scientiarum Hungaricae, 2000, T. 41, Fasc. 1/3, S. 164 . 
Die Kapellmeisterbesoldung blieb unverändert in der Höhe von $324 \mathrm{fl} \mathrm{jährlich;} \mathrm{dazu}$ bekam er 1785, wie schon im Jahre 1783, insgesamt $1800 \mathrm{fl}$ an Kostgeld für Knaben, sowies die Besoldung für zwei Altisten (Knaben) von $260 \mathrm{fl}$ und schließlich noch $57 \mathrm{fl} 36 \mathrm{x}$ für die in der Karwoche gehaltenen Metten. Das ihm vorher ausbezahlte Instruktionsgeld ging an die einzelnen Lehrer und die Besoldung von $300 \mathrm{fl}$ für die Tätigkeit in der Gnadenbildkapelle verschwand. Der Organist kam um seine Deputate (Kleider-, Mehl-, Wein- und Holzgeld, sowie das Kalkantengeld), jedoch wurde seine ordentliche Besoldung erhöht. Trotzdem bekam er jährlich $33 \mathrm{fl}$ weniger als vorher.

Das Kleidergeld wurde ebenso für alle anderen Angestellte (Sänger und Musiker) gestrichen. Die Sänger bekamen kein Rorategeld mehr, aber die Besoldung wurde einheitlich auf $190 \mathrm{fl}$ festgelegt. Den Posaunisten wurde das Geld für das Musizieren auf dem St. Stephan-Turm gekürzt, die erste drei Posaunisten erhielten seit der Regulierung $130 \mathrm{fl}$ und der vierte nur $70 \mathrm{fl}$ Auch die Gehälter der anderen Bläser wurden abgestuft - die Löhne des ersten und zweiten Kornettisten unterschieden sich um 20 fl, der Fagottist bekam nur $100 \mathrm{fl}$, wobei die zweiten Fagottisten-Stelle nach dem Tod von Jacob Payer nicht mehr besetzt worden ist.

\begin{tabular}{|c|c|c|c|c|c|c|c|}
\hline \multicolumn{8}{|c|}{ JÄHRLICHE BESOLDUNGEN DER MUSIKER BEI ST STEPHAN IN WIEN } \\
\hline \multicolumn{4}{|c|}{ Im Jahre 1783} & \multicolumn{4}{|c|}{ Im Jahre 1785} \\
\hline Anstellung & $\begin{array}{c}\text { Ordentli- } \\
\text { che Besol- } \\
\text { dung }\end{array}$ & $\begin{array}{l}\text { Gnadenbild- } \\
\text { Kapelle/ Son- } \\
\text { stiges }\end{array}$ & Insgesamt & $\begin{array}{l}\text { Ordentli- } \\
\text { che Besol- } \\
\text { dung }\end{array}$ & Sonstiges & Insgesamt & $\begin{array}{l}\text { Unter- } \\
\text { schied }\end{array}$ \\
\hline $\begin{array}{l}\text { Domkapell- } \\
\text { meister }\end{array}$ & $324 \mathrm{fl}$ & $300 \mathrm{fl}$ & $624 \mathrm{fl}$ & $324 \mathrm{fl}$ & - & $324 \mathrm{fl}$ & $-300 \mathrm{fl}$ \\
\hline Knabenlehrer & $216 \mathrm{fl}$ & - & $216 \mathrm{fl}$ & $255 \mathrm{fl}$ & - & $255 \mathrm{fl}$ & $+39 \mathrm{fl}$ \\
\hline $\begin{array}{l}\text { Knaben-Kost- } \\
\text { geld }\end{array}$ & $1400 \mathrm{fl}$ & $400 \mathrm{fl}$ & $1800 \mathrm{fl}$ & $1500 \mathrm{fl}$ & $300 \mathrm{fl}$ & $1800 \mathrm{fl}$ & $0 \mathrm{fl}$ \\
\hline Organist & $335 \mathrm{fl}$ & $200 \mathrm{fl} / 156 \mathrm{fl}$ & $691 \mathrm{fl}$ & $650 \mathrm{fl}$ & $8 \mathrm{fl}$ & $658 \mathrm{fl}$ & $-33 \mathrm{fl}$ \\
\hline 1. Bassist & $160 \mathrm{fl} 40 \mathrm{x}$ & $68 \mathrm{fl} / 12 \mathrm{fl}$ & $240 \mathrm{fl} 40 \mathrm{x}$ & $190 \mathrm{fl}$ & - & $190 \mathrm{fl}$ & $-50 \mathrm{fl} 40 \mathrm{x}$ \\
\hline 2. Bassist & $160 \mathrm{fl} 40 \mathrm{x}$ & $68 \mathrm{fl} / 12 \mathrm{fl}$ & $240 \mathrm{fl} 40 \mathrm{x}$ & $190 \mathrm{fl}$ & - & $190 \mathrm{fl}$ & $-50 \mathrm{fl} 40 \mathrm{x}$ \\
\hline 3. Bassist & $160 \mathrm{fl} 40 \mathrm{x}$ & $68 \mathrm{fl} / 12 \mathrm{fl}$ & $240 \mathrm{fl} 40 \mathrm{x}$ & $190 \mathrm{fl}$ & - & $190 \mathrm{fl}$ & $-50 \mathrm{fl} 40 \mathrm{x}$ \\
\hline 4. Bassist & - & $68 \mathrm{fl} / 5 \mathrm{fl}$ & $73 \mathrm{fl}$ & - & - & - & $-73 \mathrm{fl}$ \\
\hline 1. Tenorist & $160 \mathrm{fl} 40 \mathrm{x}$ & $68 \mathrm{fl} / 12 \mathrm{fl}$ & $240 \mathrm{fl} 40 \mathrm{x}$ & $190 \mathrm{fl}$ & - & $190 \mathrm{fl}$ & $-50 \mathrm{fl} 40 \mathrm{x}$ \\
\hline 2. Tenorist & $160 \mathrm{fl} 40 \mathrm{x}$ & $68 \mathrm{fl} / 12 \mathrm{fl}$ & $240 \mathrm{fl} 40 \mathrm{x}$ & $190 \mathrm{fl}$ & - & $190 \mathrm{fl}$ & $-50 \mathrm{fl} 40 \mathrm{x}$ \\
\hline 3. Tenorist & $160 \mathrm{fl} 40 \mathrm{x}$ & $68 \mathrm{fl} / 12 \mathrm{fl}$ & $240 \mathrm{fl} 40 \mathrm{x}$ & $190 \mathrm{fl}$ & - & $190 \mathrm{fl}$ & $-50 \mathrm{fl} 40 \mathrm{x}$ \\
\hline 4. Tenorist & - & $68 \mathrm{fl} / 5 \mathrm{fl}$ & $73 \mathrm{fl}$ & - & - & - & $-73 \mathrm{fl}$ \\
\hline $\begin{array}{l}\text { 1. Altist (Kna- } \\
\text { be) }\end{array}$ & $160 \mathrm{fl} 40 \mathrm{x}$ & $68 \mathrm{fl} / 12 \mathrm{fl}$ & $240 \mathrm{fl} 40 \mathrm{x}$ & $130 \mathrm{fl}$ & - & $130 \mathrm{fl}$ & $-110 \mathrm{fl} 40 \mathrm{x}$ \\
\hline $\begin{array}{l}\text { 2. Altist (Kna- } \\
\text { be) }\end{array}$ & $160 \mathrm{fl} 40 \mathrm{x}$ & $68 \mathrm{fl} / 12 \mathrm{fl}$ & $240 \mathrm{fl} 40 \mathrm{x}$ & $130 \mathrm{fl}$ & - & $130 \mathrm{fl}$ & -110 fl $40 x$ \\
\hline 3. Altist & $160 \mathrm{fl} 40 \mathrm{x}$ & $150 \mathrm{fl} / 12 \mathrm{fl}$ & $322 \mathrm{fl} 40 \mathrm{x}$ & $190 \mathrm{fl}$ & - & $190 \mathrm{fl}$ & $-132 \mathrm{fl} 40 \mathrm{x}$ \\
\hline $\begin{array}{l}\text { 1. Subcantor } \\
\text { [1. Choralist] }\end{array}$ & $30 \mathrm{fl}$ & $15 \mathrm{fl}$ & $45 \mathrm{fl}$ & $200 \mathrm{fl}$ & $20 \mathrm{fl}$ & $220 \mathrm{fl}$ & +175 fl \\
\hline $\begin{array}{l}\text { 2. Subcantor } \\
\text { [2. Choralist] }\end{array}$ & & $12 \mathrm{fl}$ & $12 \mathrm{fl}$ & $30 \mathrm{fl}$ & - & $30 \mathrm{fl}$ & $+18 \mathrm{fl}$ \\
\hline Choraldienste & $64 \mathrm{fl} 36 \mathrm{x}$ & $-1-$ & $64 \mathrm{fl} 36 \mathrm{x}$ & - & - & - & $-64 \mathrm{fl} 36 \mathrm{x}$ \\
\hline
\end{tabular}


Die Dommusik zu St. Stephan im 18. Jahrhundert in sozialgeschichtlicher Perspektive

\begin{tabular}{|c|c|c|c|c|c|c|c|}
\hline \multicolumn{8}{|c|}{ JÄHRLICHE BESOLDUNGEN DER MUSIKER BEI ST STEPHAN IN WIEN } \\
\hline \multicolumn{4}{|c|}{ Im Jahre 1783} & \multicolumn{4}{|c|}{ Im Jahre 1785} \\
\hline Anstellung & $\begin{array}{l}\text { Ordentli- } \\
\text { che Besol- } \\
\text { dung }\end{array}$ & $\begin{array}{l}\text { Gnadenbild- } \\
\text { Kapelle/ Son- } \\
\text { stiges }\end{array}$ & Insgesamt & $\begin{array}{l}\text { Ordentli- } \\
\text { che Besol- } \\
\text { dung }\end{array}$ & Sonstiges & Insgesamt & $\begin{array}{l}\text { Unter- } \\
\text { schied }\end{array}$ \\
\hline 1. Posaunist & $130 \mathrm{fl}$ & $68 \mathrm{fl} / 20 \mathrm{fl} 40 \mathrm{x}$ & $218 \mathrm{fl} 40 \mathrm{x}$ & $130 \mathrm{fl}$ & - & $130 \mathrm{fl}$ & $-88 \mathrm{fl} 40 \mathrm{x}$ \\
\hline 2. Posaunist & $130 \mathrm{fl}$ & $68 \mathrm{fl} / 20 \mathrm{fl} 40 \mathrm{x}$ & $218 \mathrm{fl} 40 \mathrm{x}$ & $130 \mathrm{fl}$ & - & $130 \mathrm{fl}$ & $-88 \mathrm{fl} 40 \mathrm{x}$ \\
\hline 3. Posaunist & $130 \mathrm{fl}$ & $68 \mathrm{fl} / 20 \mathrm{fl} 40 \mathrm{x}$ & $218 \mathrm{fl} 40 \mathrm{x}$ & $130 \mathrm{fl}$ & - & $130 \mathrm{fl}$ & $-88 \mathrm{fl} \mathrm{40x}$ \\
\hline 4. Posaunist & - & $68 \mathrm{fl} /-$ & $68 \mathrm{fl}$ & $70 \mathrm{fl}$ & - & $70 \mathrm{fl}$ & $+2 \mathrm{fl}$ \\
\hline 1. Fagottist & $130 \mathrm{fl}$ & $68 \mathrm{fl} / 20 \mathrm{fl} 40 \mathrm{x}$ & $218 \mathrm{fl} 40 \mathrm{x}$ & $100 \mathrm{fl}$ & - & $100 \mathrm{fl}$ & $-118 \mathrm{fl} 40 \mathrm{x}$ \\
\hline 2. Fagottist & - & $68 \mathrm{fl} /-$ & $68 \mathrm{fl}$ & - & - & - & $-68 \mathrm{fl}$ \\
\hline 1. Kornettist & $160 \mathrm{fl}$ & $68 \mathrm{fl} /-$ & $228 \mathrm{fl}$ & $150 \mathrm{fl}$ & - & $150 \mathrm{fl}$ & $-78 \mathrm{fl}$ \\
\hline 2. Kornettist & $140 \mathrm{fl}$ & $54 \mathrm{fl} /-$ & $194 \mathrm{fl}$ & $130 \mathrm{fl}$ & - & $130 \mathrm{fl}$ & $-64 \mathrm{fl}$ \\
\hline 1. Violinist & $250 \mathrm{fl}$ & $54 \mathrm{fl} /-$ & $304 \mathrm{fl}$ & $250 \mathrm{fl}$ & - & $250 \mathrm{fl}$ & $-54 \mathrm{fl}$ \\
\hline 2. Violinist & $150 \mathrm{fl}$ & $50 \mathrm{fl} /-$ & $200 \mathrm{fl}$ & $225 \mathrm{fl}$ & - & $225 \mathrm{fl}$ & $+25 \mathrm{fl}$ \\
\hline 3. Violinist & $225 \mathrm{fl}$ & $-1-$ & $225 \mathrm{fl}$ & $225 \mathrm{fl}$ & - & $225 \mathrm{fl}$ & $0 \mathrm{fl}$ \\
\hline 4. Violinist & $225 \mathrm{fl}$ & $-1-$ & $225 \mathrm{fl}$ & $225 \mathrm{fl}$ & - & $225 \mathrm{fl}$ & $0 \mathrm{fl}$ \\
\hline 5. Violinist & - & $154 \mathrm{fl} /-$ & $154 \mathrm{fl}$ & $150 \mathrm{fl}$ & - & $150 \mathrm{fl}$ & $-4 \mathrm{fl}$ \\
\hline 6. Violinist & - & $120 \mathrm{fl} /-$ & $120 \mathrm{fl}$ & $120 \mathrm{fl}$ & - & $120 \mathrm{fl}$ & $0 \mathrm{fl}$ \\
\hline 7. Violinist & $104 \mathrm{fl}$ & $-1-$ & $104 \mathrm{fl}$ & $100 \mathrm{fl}$ & - & $100 \mathrm{fl}$ & $-4 \mathrm{fl}$ \\
\hline 8. Violinist & - & $100 \mathrm{fl} /-$ & $100 \mathrm{fl}$ & $100 \mathrm{fl}$ & - & $100 \mathrm{fl}$ & $0 \mathrm{fl}$ \\
\hline 9. Violinist & $32 \mathrm{fl}$ & $-/-$ & $32 \mathrm{fl}$ & $36 \mathrm{fl}$ & - & $36 \mathrm{fl}$ & $+4 \mathrm{fl}$ \\
\hline 10. Violinist & $32 \mathrm{fl}$ & $-/ 52 \mathrm{fl}$ & $84 \mathrm{fl}$ & $32 \mathrm{fl}$ & - & $32 \mathrm{fl}$ & $-52 \mathrm{fl}$ \\
\hline 11. Violinist & $32 \mathrm{fl}$ & $-/ 52 \mathrm{fl}$ & $84 \mathrm{fl}$ & $32 \mathrm{fl}$ & - & $32 \mathrm{fl}$ & $-52 \mathrm{fl}$ \\
\hline 12. Violinist & $19 \mathrm{fl} 12 \mathrm{x}$ & $-/-$ & $19 \mathrm{fl} 12 \mathrm{x}$ & $20 \mathrm{fl}$ & - & $20 \mathrm{fl}$ & $+48 x$ \\
\hline 1. Violoncellist & $250 \mathrm{fl}$ & $-1-$ & $250 \mathrm{fl}$ & $250 \mathrm{fl}$ & - & $250 \mathrm{fl}$ & $0 \mathrm{fl}$ \\
\hline $\begin{array}{l}\text { 2. Violoncel- } \\
\text { list }\end{array}$ & $136 \mathrm{fl}$ & $-1-$ & $136 \mathrm{fl}$ & $68 \mathrm{fl}$ & - & $68 \mathrm{fl}$ & $-68 \mathrm{fl}$ \\
\hline 1. Violonist & $150 \mathrm{fl}$ & $68 \mathrm{fl} /-$ & $218 \mathrm{fl}$ & $150 \mathrm{fl}$ & - & $150 \mathrm{fl}$ & $-68 \mathrm{fl}$ \\
\hline 2. Violonist & - & $50 \mathrm{fl} /-$ & $50 \mathrm{fl}$ & $50 \mathrm{fl}$ & - & $50 \mathrm{fl}$ & $0 \mathrm{fl}$ \\
\hline Hoftrompeter ${ }^{49}$ & - & $\begin{array}{c}147 \mathrm{fl} \\
12 x / 34 \mathrm{fl}\end{array}$ & $181 \mathrm{fl} 12 \mathrm{x}$ & $120 \mathrm{fl}$ & - & $120 \mathrm{fl}$ & -61 fl 12x \\
\hline $\begin{array}{l}\text { Gesamte } \\
\text { Musik }\end{array}$ & - & $-/ 119$ fl $12 x$ & 119 fl $12 x$ & - & $19 \mathrm{fl} 12 \mathrm{x}$ & $19 \mathrm{fl} 12 \mathrm{x}$ & $-100 \mathrm{fl}$ \\
\hline Insgesamt: & & & $\begin{array}{c}9.865 \mathrm{fl} \\
52 \mathrm{x}\end{array}$ & & & $7.889 \mathrm{fl} 12 \mathrm{x}$ & -1.976 fl $40 x$ \\
\hline
\end{tabular}

Diese Tabelle gibt einen Einblick in den Verlauf und die finanziellen Auswirkungen der Umstrukturierung, aber auch in die vorherigen Strukturen, der Dommusik. Vor allen am Beispiel der Streicher werden die Verbindungen zwischen Essential- und Gnadenbildkapelle sichtbar. Dank der Nummerierung der einzelnen Posten geht hervor, dass die Violinisten in der Gnadenbildkapelle die Hoffnung hatten, durch das Vorrücken eine Anstellung als Essentialist zu erhalten. Musterhaft ist dabei die Kariere des fünften Violinisten in der Zeit der Musikregulierung Otto Heinrich Ponheimer. 1761 begann er seine Karriere als

49 Die Zahlungen an den kaiserlichen Hoftrompeter unterschieden sich jedes Jahr vor und nach der Regulierung, je nach der Menge der geleisteten Dienste. 
Vestenburglicher Musicus ${ }^{50}$ [Violinist] mit einer Besoldung von $40 \mathrm{fl}$ an. 1765 rückte er an die Stelle des verstorbenen Joseph Gämbroth vor, wobei er mit $100 \mathrm{fl}$ jährlich entlohnt wurde. Im Jahre 1771 wurde sein Gehalt um 4 fl erhöht. Ab 1779 ist er als Mitglied der Gnadenbild-Kapelle geführt und somit verbesserte sich sein Einkommen auf $120 \mathrm{fl}$ jährlich. ${ }^{51}$ Nach dem Tod von Franz Ignaz Braun im Jahre 1780 wurde er durch ein Magistratsdekret zum 5. Violinisten mit $154 \mathrm{fl}$ jährlich ernannt, immer noch als Mitglied der Gnadenbildkapelle. ${ }^{52}$ Als solcher verblieb er bis ins Jahr 1783; 1784 wurden alle Musiker, die ausschließlich zur Gnadenbildkapelle gehörten, der Essentialkapelle zugeschrieben und nach der Besoldungsregulierung wurde sein Gehalt auf $150 \mathrm{fl}$ niedergesetzt. Auf der anderen Seite verlor er, sowie die anderen Musiker, dank dieser Umstrukturierung nach der Einstellung des Musikbetriebs beim Altar des Gnadenbilds Maria Pötsch seine Stelle nicht.

\section{Beispielfälle: Versuche um Verbesserung der finanziellen Lage}

Die schwierige finanzielle Situation der Musiker illustrieren im Aktenmaterial erhaltene Vorschläge und Bitten um verschiedene Zuschüsse. Da die Reform auch Deputate betraf, bat der Domorganist Matthäus Mittlmayer am 19. Juli 1785 um Erneuung des jährlichen Holzgeldes (21 fl $36 \mathrm{x}$ ), welches ihm bei seiner Anstellung verliehen, seit der Musikreform allerdings nicht mehr ausbezahlt wurde. Nach der Musikreform wurde ihm die Gebühr entnommen, wobei dem Kapellmeister sein Holzgeld belassen wurde. Sein Gesuch wurde abgewiesen, weil erstens der Kapellmeister eine besondere Stellung einnahm und zweitens, weil nach einer Bewilligung andere Musiker ähnliche Forderungen stellen hätten können. ${ }^{53}$ Daraus ergibt sich, dass der Domkapellmeister sein Holzdeputat weiter bekam, obwohl es nicht in den Ausgaben erfasst ist.

Im August 1785 wurden an den Magistrat mehrere Briefe, sowohl von Musikern als auch von Sängern geschickt. Am 19. Juli ist ein Gesuch der sechs Vokalisten um eine Verbesserung ihrer Besoldungen an den Magistrat gesendet worden. Den Vokalisten wurde das Kleidergeld, das Geld für das Abholen Sängerknaben, sowie die Bezahlung für kirchenmeisterliche Requiem entzogen. Die verschiedenen Musikdienste im Dom, sowie die vorherigen Pflichten der Musiker wurden stark reduziert. Das Completorium wurde beispielsweise Teil von Vespern, der Circuitus gehörte nun zum Hochamt. Sie verloren auch die Besoldung der Gnadenbildkapelle. Deshalb wurde den Bittstellern $30 \mathrm{fl}$ zu ihren Gehältern zugesprochen und zugelegt, was insgesamt ihr bisheriges Gehalt erhöhte. Insgesamt bekam jeder Vokalist 1785 eine Besoldung von insgesamt $400 \mathrm{fl}$, wovon es

50 Siehe Kapitel: Finanzierungsquellen der Dommusik. In KRAMÁŘOVÁ, Helena. Die Dommusik zu St. Stephan im 18. Jahrhundert - Institutionsgeschichte. In Musicologica Brunensia, 54, 2019, Nr. 1, S. 137-171.

51 Vgl. AT-DAWST, KMA, Rechnungsbuch 1779, Fol. 111r.

52 Es wäre falsch, anzunehmen, dass sich Reinhards finanzielle Lage mit dem Vorrücken von der Gnadenbildkapelle verschlechterte. Die ordentliche Besoldung betrug zwar $100 \mathrm{fl}$ jährlich, doch dazu kam noch Geld von Ausgaben, die für die gesamte Musik bestimmt wurde (Stiftungen, Jahrestage, etc.).

53 Vgl. AT-DAWST, KMA, Erledigungen 1771-1786, Kart. 7/2, Fol. 481. 
sich ihrer Meinung nach allerding nicht leben ließ. Aus dem Vergleich mit der oben angeführten Tabelle der offiziellen Besoldungen wird ersichtlich, dass die Deputathöhe der Sänger ca. $240 \mathrm{fl}$ betragen musste; darunter war z. B. das Einkommen durch Begräbnisse. Für die Kirchenkasse wäre eine weitere Besoldungsvermehrung eine große Belastung gewesen, wie vom Kirchenmeisteramt behauptet wurde. Da ein Teil der Musiker in der Hofkapelle wirkte und ein anderer Teil in anderen Kirchen angestellt war, wurde die Entscheidung getroffen, den Vokalisten keine zusätzliche Besoldung zu bewilligen. ${ }^{54}$

Auch der in der Domkapelle angestellte Altist Anton Pacher stellte einen Antrag auf eine Zulage zu seiner Besoldung, die nach der Regulierung $190 \mathrm{fl}$ betrug. Er argumentierte, dass er bereits 33 Jahre bei der Domkirche diene und deswegen seine AltistenStellen bei St. Michael und St. Peter verlassen habe, um eifrig und ausschließlich bei St. Stephan dienen zu können. Vorher erhielt er $150 \mathrm{fl}$ in der Gnadenbildkapelle und dazu $130 \mathrm{fl}$ als Essentialist, wozu noch das Kleidergeld, Corporis Christi, Vestenburgliche Stiftung und Rorategeld kam. Ab 1784 bekam er insgesamt nur $190 \mathrm{fl}$, wovon er seine Frau und vier unmündige Kinder ernähren musste. Er verglich seine Besoldung mit der Taxe für einen Kapellknaben, die $300 \mathrm{fl}$ betrug. Sein Gesuch wurde gleich wie die anderen abgewiesen. Ihm wurde geantwortet, dass er nicht gezwungen war, seine Anstellung in den zwei genannten Kirchen zu verlassen und, auch wenn er diese Stellen innehätte, sollte dies seine Eifrigkeit im Dom keineswegs negativ beeinflussen. Obwohl der Magistrat annehmen konnte, dass die Versorgung seiner Familie wohl schwierig war, wurde betont, dass seine vorherige Essential-Besoldung von $130 \mathrm{fl}$ nicht gekürzt wurde und er anstatt der Gnadenbild-Besoldung eine Zulage von $60 \mathrm{fl}$ erhielt. Das Kleidergeld sowie Rorategeld könne er nicht mehr bekommen, da die Rorate allein von der Orgel begleitet werde. Die Vestenburgliche Stiftung wurde ebenfalls einbehalten. Von daher hatte die Kirche keine andere Möglichkeit für Verbesserung der Musiker-Besoldungen..$^{55}$ Ebenso versuchten auch andere Musiker ihr Glück und stellten verschiedene Anträge mit der Hoffnung auf Verbesserung ihrer Lebensumstände und auf Erhöhung ihres Lebensunterhalts, was wie bei den vorher erwähnten Gesuchen abgelehnt wurde. ${ }^{56}$

Die Tendenzen bezüglich der Besoldung von Kirchenmusikern gingen in den späteren Jahren eher in die andere Richtung; es wurde versucht, so viel wie möglich zu sparen. Dieses Vorgehen veranschaulicht der Vorschlag der Reduzierung von Violinisten in der Domkapelle aus dem Jahre 1787. Als der dritte Violinist Joseph Adam verstarb, baten die anderen Violinisten um gewöhnliches Vorrücken. ${ }^{57}$ Im Brief des Kirchenverwalters wurde wegen der Regulierung der Musik und der damit verbundenen erschwerten Lebensbedingungen der Musiker vorgeschlagen, dass sich bei einem Todesfalle die Anzahl der Violinisten vermindert und die Besoldung des Verstorbenen auf diese verteilt werden solle. Es

54 Vgl. AT-DAWST, KMA, Erledigungen 1771-1786, Kart. 7/2, Fol. 483.

55 Vgl. Ebenda, Fol. 482.

56 Zu nennen sei das Gesuch von Tobias Gsur (Vgl. AT-DAWST, KMA, Erledigungen 1771-1786, Kart. 7/2, Fol. 485), von den Tenoristen Leopold Panschab (Vgl. Ebenda, Fol. 486) oder Joseph Kammermeyer, Violonisten (Vgl. Ebenda, Fol. 473).

57 Dies geht aus den folgenden Akten hervor: Vgl. AT-DAWST, KMA, Erledigungen 1786-1792, Kart. 7a/1, Fol. 542-554. 
sei bei der Musikreform mündlich veranlasset worden, dass von den ursprünglich zwölf Violinistenstellen künftig nur mehr sechs besetzt werden und die höheren Besoldungen vermindert werden sollen. Diese mündliche Vereinbarung sollte laut des unterschriebenen Kirchenverwalters eingehalten werden und er selbst wäre ebenfalls dafür, die ViolinistenStellen auf sechs zu kürzen und dabei noch zwei Besoldungsklassen zu bilden - die ersten drei Violinisten würden $150 \mathrm{fl}$ jährlich bekommen und die anderen drei $130 \mathrm{fl}$, weil jeder Violinist, aber auch Violonist und Violoncellist, bei der neugeregelten Ordnung das ganze Jahr hindurch nicht mehr als höchstens 65 Tage seinen Dienst verrichten musste. Deshalb glaube der Kirchenverwalter, dass für jeden Dienst der ersten drei Violinisten 2 fl $20 \mathrm{x}$ und für die drei übrigen $2 \mathrm{fl}$ zu bestimmen wären. Wenn der Vorschlag angenommen würde, so könne sich Herr Ponheimer keine weitere Hoffnung mehr machen, in eine höhere Besoldung zu kommen, wohl aber könnte die Besoldung folglich ausschauen:

\begin{tabular}{|c|c|c|c|c|c|}
\hline \multicolumn{6}{|c|}{ Violinisten-Gehälter und Vorschlag der Kostenreduzierung } \\
\hline \multicolumn{2}{|c|}{$\begin{array}{c}\text { Ursprüngliche } \\
\text { Besoldungen }(1787)^{58}\end{array}$} & \multicolumn{2}{|l|}{ Besoldungsvorschlag ${ }^{59}$} & \multicolumn{2}{|c|}{ Stand im Jahre $1792^{60}$} \\
\hline 1. Violinist & $250 \mathrm{fl}$ & $\begin{array}{l}\text { 1. Violinist - Anton Hofmann, altes } \\
\text { Gehalt }\end{array}$ & $250 \mathrm{fl}$ & $\begin{array}{l}\text { Violinist - Anton Hof- } \\
\text { mann }\end{array}$ & $250 \mathrm{fl}$ \\
\hline 2. Violinist & $225 \mathrm{fl}$ & $\begin{array}{l}\text { 2. Violinist - Niclas Reinhard, altes } \\
\text { Gehalt }\end{array}$ & $225 \mathrm{fl}$ & $\begin{array}{l}\text { Violinist - Niclas Rein- } \\
\text { hard }\end{array}$ & $225 \mathrm{fl}$ \\
\hline 3. Violinist & $225 \mathrm{fl}$ & 3. Violinist - Markus Hofer & $225 \mathrm{fl}$ & $\begin{array}{l}\text { Violinist - Otto Pon- } \\
\text { heimer }\end{array}$ & $225 \mathrm{fl}$ \\
\hline 4. Violinist & $225 \mathrm{fl}$ & $\begin{array}{l}\text { 4. Violinist - Otto Ponheimer nach } \\
\text { neuen Fuß }\end{array}$ & $150 \mathrm{fl}$ & Violinist - Anton Borghi & $225 \mathrm{fl}$ \\
\hline 5. Violinist & $150 \mathrm{fl}$ & $\begin{array}{l}\text { 5. Violinist - Anton Borghi hatte } \\
120 \mathrm{fl} \text {, künftig aber }\end{array}$ & $140 \mathrm{fl}$ & $\begin{array}{l}\text { Violinist - Joseph } \\
\text { Klemp }\end{array}$ & $150 \mathrm{fl}$ \\
\hline 6. Violinist & $120 \mathrm{fl}$ & $\begin{array}{l}\text { 6. Violinist-Michael Hofer hatte } \\
100 \mathrm{fl} \text {, künftig aber }\end{array}$ & $120 \mathrm{fl}$ & $\begin{array}{l}\text { Violinist - Johann } \\
\text { Scheidl }\end{array}$ & $120 \mathrm{fl}$ \\
\hline 7. Violinist & $100 \mathrm{fl}$ & $\begin{array}{l}\text { 7. Violinist - anstatt H: Mechtl, } \\
\text { Joseph Klemp dieser hatte bishero } \\
36 \mathrm{fl} \text { - künftig }\end{array}$ & $70 \mathrm{fl}$ & Violinist - Franz Hofer & $100 \mathrm{fl}$ \\
\hline 8. Violinist & $100 \mathrm{fl}$ & $\begin{array}{l}\text { 8. Violinist - Johann Scheidl hatte } \\
\text { bishero } 36 \mathrm{fl} \text { künftig } \\
\end{array}$ & $60 \mathrm{fl}$ & $\begin{array}{l}\text { Violinist - Leopold } \\
\text { Reinhard } \\
\end{array}$ & $36 \mathrm{fl}$ \\
\hline 9. Violinist & $36 \mathrm{fl}$ & $\begin{array}{l}\text { 9. Violinist - Mathias Hofer hatte } \\
\text { bishero } 32 \text { fl künftig }\end{array}$ & $50 \mathrm{fl}$ & $\begin{array}{l}\text { Violinist - Joseph Lor- } \\
\text { ber (bis Ende Juni) }\end{array}$ & $18 \mathrm{fl}$ \\
\hline 10. Violinist & $36 \mathrm{fl}$ & $\begin{array}{l}\text { 10. Violinist - Franz Hofer hatte } 20 \\
\mathrm{fl} \text { - künftig }\end{array}$ & $40 \mathrm{fl}$ & $\begin{array}{l}\text { Violinisten Accessist - } \\
\text { Franz Kammermayer }\end{array}$ & $50 \mathrm{fl}$ \\
\hline 11. Violinist & $32 \mathrm{fl}$ & - & - & $\begin{array}{l}\text { Violinist - Mathias } \\
\text { Altmüller }\end{array}$ & $36 \mathrm{fl}$ \\
\hline 12. Violinist & $20 \mathrm{fl}$ & - & - & $\begin{array}{l}\text { Violinist - Karl Ponhei- } \\
\text { mer für die letzte } 4 \text { Mo- } \\
\text { nate von jährlich } 36 \mathrm{fl}\end{array}$ & $12 \mathrm{fl}$ \\
\hline Insgesamt & $1.519 \mathrm{fl}$ & & $1.330 \mathrm{fl}$ & & $1.447 \mathrm{fl}$ \\
\hline
\end{tabular}

58 Vgl. AT-DAWST, KMA, Erledigungen 1786-1792, Kart. 7a/1, Fol. 555.

59 Ebenda, Kart. 7a/1, Fol. 555.

60 Exzerpte nach: AT-WStLA, Handschriften, A41/29 Kirchenmeisteramts-Rechnungen von St. Stephan -1792, S. 63. 
Weiters ist hinzuzufügen, dass diese Veränderung erst ab Oktober zum Ausdruck kommen konnte, da die Witwen des verstorbene Violinisten Joseph Adam, sowie des Violinisten Fridrich Mechtl, den Anspruch auf Sterbequartal hatten. ${ }^{61}$ Von Kirchenmeister Furthmoser wurde noch deutlich gemacht, dass die Ersparnisse zwar nur $189 \mathrm{fl} \mathrm{jährlich} \mathrm{sein}$ werden, diese jedoch künftig - nach dem Tod von Anton Hofmann, Niklas Reinhard und Markus Hofer - für die Kirchenkasse bedeutend sein würden, da nach ihrem Tod die Anzahl der Violinisten auf die genannten sechs herabgesetzt und die Besoldungen auf $150 \mathrm{fl}$ und $130 \mathrm{fl}$ gesenkt würden - somit werde die Kirchenkasse $679 \mathrm{fl} \mathrm{mehr}$ zur Verfügung hätten.

Die von ihm vorgeschlagene Lösung der Violinistenbesoldung wurde zwar durchgeführt - im Rechnungsbuch aus dem Jahre 1787 wurden die Gehälter von Violinisten ab Oktober neu verrechnet. Die tatsächlichen Ausgaben für die Violinisten (inkl. der Sterbequartale für Witwen, usw.) betrugen im Jahre 1787 insgesamt $1480 \mathrm{fl}$. Da die danach kommenden Jahrgänge leider nicht mehr erhalten sind, kann erst auf das Jahr 1792 zurückgegriffen werden.

Vergleicht man die Zahlen der Tabelle, so fällt auf, dass zwar einige Veränderungen durchgeführt wurden, das Vorrücken der Violinisten und die von ihnen jahrelang angestrebte Gehaltsverbesserung jedoch immer wieder erlaubt wurde. Dies wird anhand des Falls von Otto Ponheimer deutlich - der Kirchenmeister behauptete 1787, dass er sich keine Hoffnungen diesbezüglich machen solle, dennoch wurde er fünf Jahre später als dritter Violinist mit $225 \mathrm{fl}$ besoldet. Die deklarierten Gesamtersparnisse, zu denen das neu aufgestellte System führen sollte, traten nicht ein. ${ }^{62}$

Danach finden sich die nächsten Versuche um Verbesserung bei der Bezahlung erst am Anfang der 90er Jahre. Im Mai 1794 beantragte der erste Posaunist Johann Adlmann eine Besoldungserhöhung für die sechs Instrumentalisten für ihre Tätigkeit in der Karwoche. Seinen Angaben nach wurde den Instrumentalisten für das Spielen in der Karwoche vor der Musikreform $12 \mathrm{fl}$ gegeben, dieses Geld sei jedoch ab 1784 in die reguläre Besoldung eingegliedert worden. Der Kirchenmeister hob hervor, dass die Zeremonien zwar ohne Posaunisten stattfinden könnten, es bei einer Domkirche jedoch erforderlich sei, diese musikalischen Aufgaben, vor allem in der Karwoche, zu erfüllen, und darüber hinaus extra zu bezahlen, weil diese Musiker überhaupt sehr bedürftig und arm seien und der Verlust von $12 \mathrm{fl} \mathrm{sehr}$ beträchtlich sei. ${ }^{63}$

Die Vokalisten baten im Juni 1794 wieder um eine Zulage; nach dem Tod des Bassisten Tobias Gsur rückte der bisherige Posaunist Joseph Hofmann an dessen Stelle vor. Im Zuge dessen kam es zum Vorschlag, die Posaunisten-Stelle nicht neu zu besetzen und das Gehalt unter den Bittstellern zu verteilen. Der Vorschlag wurde diskutiert und das Resultat war, nach Joseph Hoffmann keinen weiteren Posaunisten anzustellen, das Geld

61 Vgl. AT-DAWST, KMA, Erledigungen 1786-1792, Kart. 7a/1, Fol. 555.

62 Im Jahre 1793 betrugen die Gesamtkosten für Violinisten $1.406 \mathrm{fl} 34 \mathrm{x}$ und ein Jahr danach, in 1794, $1.383 \mathrm{fl}$.

63 Vgl. AT-DAWST, KMA, Erledigungen 1792-1800, Kart. 8/1, Fol. 764. 
jedoch nicht unter den anderen Vokalisten zu verteilen, sondern in die Kirchenkasse überzuleiten. ${ }^{64}$

All diese Beispiele zeigen, dass das Spannungsfeld zwischen dem Bedürfnis nach musikalischer Feierlichkeit der Liturgie und den Musikkosten in den 80er und 90er Jahren des 18. Jahrhunderts ein nicht leicht zu lösendes Problem darstellte. Wie sich die finanzielle Stellung der Musiker und die Lage der Domkapelle nun tatsächlich weiter entwickelte kann erst im Rahmen weiterer Forschung festgestellt werden.

\section{Resümee}

Unter den kirchlichen Institutionen in Wien stellte die Anstellung bei der Domkirche zu St. Stephan eine Möglichkeit für Musiker und Sänger dar, ihren Lebensunterhalt und somit auch die Versorgung ihrer Familien zu sichern.

Anhand des erforschten Materials zeigt sich, dass für die Domkirche nicht nur unbezahlte Adjunkten wichtig waren, sondern auch, dass es eine Art Warteliste für den Erhalt einer festen Anstellung im Stephansdom gab, wofür die Vorgehensweise des Vorrückens von Bedeutung war. Durch das Vorrücken verbesserte sich die finanzielle Lage der Musiker, sowie deren Stellung in der Dommusik. Durch ein Dekret aus 1722 wurde den Angestellten versichert, dass die hinterlassene Familie eine Dienstleistung in Form eines Quartalgehaltes erhält. Berücksichtigt wurde auch die Versorgung der Sängerknaben im 18. Jahrhundert und die wichtigsten Veränderungen im Knabenunterricht.

Diese Prozesse weisen auf eine über lange Zeit unverändert gebliebene Tradition hin, die teilweise durch die Gottesdienstreform Kaiser Josephs II. gebrochen wurden. Durch die Reduktion der Gottesdienste und die strenge Regulierung der dabei gespielten Musik verminderten sich die Gelegenheiten, bei denen das gesamte Ensemble spielen konnte. Außerdem wurde die Gnadenbildkapelle komplett aufgehoben, weil die finanziellen Mittel nicht mehr zur Verfügung standen. Trotzdem konnten alle Musiker, die ausschließlich zur Gnadenbildkapelle gehörten, dank einer Umstrukturierung als Dommusiker weiterhin angestellt bleiben. Verminderte Gehälter, sowie erschwerte Lebensverhältnisse führten zu verschiedenen Vorschlägen und Bitten um Verbesserung der Besoldungen, was durch ausgewählte Beispiele illustriert wurde.

Inwiefern die Beschäftigung der im Stephansdom angestellten Musiker und Sänger, ihre Sicherheiten und Pflichten, einen speziellen Fall darstellen, muss natürlich durch weitere Forschungen genauer betrachtet werden. Trotzdem kann die Musikpflege im Stephansdom als ein wichtiges Vergleichsmodell für andere Residenz- und Haupt- und Königlichen Städte in den Habsburgischen Gebieten gesehen werden.

64 Vgl. AT-DAWST, KMA, Erledigungen 1792-1800, Kart. 8/1, Fol. 761; diese Vorgehensweise ist ebenfalls in den Rechnungsbüchern aus dem Jahr 1794 nachvollziehbar. Vgl. AT-DAWST, KMA, Rechnungsbuch 1794, S. 64 . 


\section{Bibliography}

\section{Sources}

Archiv města Brna (AMB) [Archiv der Stadt Brünn], Bestand A1/12 Spisovna in publicis sine signo, Inv.-Nr. 3023, nefol. Plan / Einer Verhältnußmessigen Eintheilung zwischen / den allerhöchst bestimmten drey Pfarreyen der / k. Haupt Stadt Brünn.

Domarchiv St. Stephan (weiter: AT-DAWST), Kirchenmeisteramt von St. Stephan (weiter: KMA), Kirchenrechnung 1721.

AT-DAWST, KMA, Kirchenrechnung 1722.

AT-DAWST, KMA, Kirchenrechnung 1732.

AT-DAWST, KMA, Kirchenrechnung 1743.

AT-DAWST, KMA, Kirchenrechnung 1747.

AT-DAWST, KMA, Kirchenrechnung 1777.

AT-DAWST, KMA, Kirchenrechnung 1779.

AT-DAWST, KMA, Kirchenrechnung 1784.

AT-DAWST, KMA, Kirchenrechnung 1794.

AT-DAWST, KMA, Erledigungen 1786-1792, Kart. 7a/1, Fol. 542-554.

AT-DAWST, KMA, Erledigungen 1786-1792, Kart. 7a/1, Fol. 555.

AT-DAWST, KMA, Erledigungen 1771-1786, Kart. 7/2, Fol. 402.

AT-DAWST, KMA, Erledigungen 1771-1786, Kart. 7/2, Fol. 442.

AT-DAWST, KMA, Erledigungen 1771-1786, Kart. 7/2, Fol. 473.

AT-DAWST, KMA, Erledigungen 1771-1786, Kart. 7/2, Fol. 481.

AT-DAWST, KMA, Erledigungen 1771-1786, Kart. 7/2, Fol. 482.

AT-DAWST, KMA, Erledigungen 1771-1786, Kart. 7/2, Fol. 482

AT-DAWST, KMA, Erledigungen 1771-1786, Kart. 7/2, Fol. 485-486.

AT-DAWST, KMA, Erledigungen 1792-1800, Kart. 8/1, Fol. 727.

AT-DAWST, KMA, Erledigungen 1792-1800, Kart. 8/1, Fol. 759.

AT-DAWST, KMA, Erledigungen 1792-1800, Kart. 8/1, Fol. 761.

AT-DAWST, KMA, Erledigungen 1792-1800, Kart. 8/1, Fol. 764

AT-DAWST, KMA, Erledigungen 1792-1800, Kart. 8/2, Fol. 807-808.

AT-DAWST, KMA, Erledigungen 1800-1804, Kart. 9/1, Fol. 1005.

AT-DAWST, KMA, Erledigungen 1800-1804, Kart. 9/1, Fol. 1051.

AT-DAWST, KMA, Erledigungen 1800-1804, Kart. 9/1, Fol. 1100.

AT-DAWST, KMA, Erledigungen 1800-1804, Kart. 9/2, Fol. 1146.

AT-DAWST, KMA, Erledigungen 1804-1808, Kart. 10/1, Fol. 1202.

AT-DAWST, KMA, Erledigungen 1804-1808, Kart. 10/1, Fol. 1272.

AT-DAWST, KMA, Erledigungen 1804-1808, Kart. 10/2, Fol. 1304.

AT-DAWST, KMA, Erledigungen 1804-1808, Kart. 10/2, Fol. 1316.

AT-DAWST, KMA, Erledigungen 1808-1811, Kart. 11/1, Fol. 1461.

AT-DAWST, KMA, Erledigungen 1808-1811, Kart. 11/1, Fol. 1446.

AT-DAWST, KMA, Erledigungen 1808-1811, Kart. 11/1, Fol. 1450.

AT-DAWST, KMA, Erledigungen 1808-1811, Kart. 11/1, Fol. 1453. 
Wiener Stadt- und Landesarchiv (weiter: AT-WStLA), Handschriften A41/20 KirchenmeisteramtsRechnungen von St. Stephan -1702.

AT-WStLA, Handschriften A41/29 Kirchenmeisteramts-Rechnungen von St. Stephan -1792.

AT-WStLA, Hauptarchiv A1/M14, Wolfgang Amadeus Mozart 5, Gesuch um Verleihung der Kapellmeistersadjunktenstelle.

\section{Literature}

BIBA, Otto. Der Sozialstatus des Musikers. In Joseph Haydn in seiner Zeit. Eisenstadt 1982, S. 105-113.

BIBA, Otto. Die Wiener Kirchenmusik um 1783. In Beiträge zur Musikgeschichte des 18. Jahrhunderts (I/2). Eisenstadt: Institut für Österreichische Kulturgeschichte, 1971, S. 7-67.

HILSCHER, Elisabeth. Musikbruderschaften als Karrierenetzwerke für Stadt und Hof in Wien. In HILSCHER, Elisabeth Th. Wiener Tonkünstler-Sozietät. In Oesterreichisches Musiklexikon online [online], 20XX. [cit. 2019-05-13] URL: 〈https://www.musiklexikon.ac.at/ml/musik_T/Tonkuenstlersozietaet.xml>.

HOCHRADNER, Thomas - VÖSÖMARTY, Géza Michael. Zur Musikpflege am Altar Mária Pócs (Maria Pötsch) in St. Stephan in Wien. In Studia Musicologica Academiae Scientiarum Hungaricae, 2000, T. 41, Fasc. 1/3, S. 156-164.

LOBENWEIN, Elisabeth - SCHEUTZ, Martin - WEISS, Alfred Stefan (Hg.). Bruderschaften als multifunktionale Dienstleister der Frühen Neuzeit in Zentraleuropa. Wien: Böhlau, 2018, S. 357-372.

KRAMÁŘOVÁ, Helena. Die Dommusik zu St. Stephan im 18. Jahrhundert - Institutionsgeschichte. In Musicologica Brunensia, 54, 2019, Nr. 1, S. 137-171.

SALMEN, Salmen. Der Sozialstatus des Berufsmusikers vom 17. bis 19. Jahrhundert. Kassel: BärenreiterVerlag, 1971.

SEHNAL, Jiří. Hudba v olomoucké katedrále v 17. a 18. století. Brno: Moravské muzeum, 1988. 